\title{
Microlocal Reduction of Ordinary Differential Operators with a Large Parameter
}

\author{
By \\ Takashi AOKI* and Jun-ichi YosHIDA**
}

\section{§ 0. Introduction}

The purpose of this paper is to generalize the results of [2] and to discuss exact WKB analysis for differential equations of second order with a large parameter $\eta$ from the view point of the transformation theory of differential operators. In $[2]$, it is shown that a WKB solution of the equation

$$
\left(\frac{d^{2}}{d \tilde{x}^{2}}-\eta^{2} Q(\tilde{x})\right) \tilde{\varphi}(\tilde{x}, \eta)=0
$$

around a simple zero of $Q$ can be transformed to a WKB solution of the Airy equation with the large parameter

$$
\left(\frac{d^{2}}{d x^{2}}-\eta^{2} x\right) \varphi(x, \eta)=0,
$$

where $Q$ is a holomorphic function. That is, for the Borel transform $\tilde{\varphi}_{B}(\tilde{x}, y)$ and $\varphi_{B}(x, y)$ of $\phi(\tilde{x}, \eta)$ and $\varphi(x, \eta)$ respectively, there exist a local coordinate $x=x(\tilde{x})$ and an invertible microdifferential operator $T$ satisfying $\tilde{\varphi}_{B}(\tilde{x}, y)$ $=\left.T \varphi_{B}(x, y)\right|_{x=x(\tilde{x})}$.

Taking Borel transformation of (0.1) and (0.2), we see that $\widetilde{\varphi}_{B}$ and $\varphi_{B}$ satisfy the following partial differential equations :

Communicated by T. Kawai, July 13, 1992. Revised February 23, 1993.

1991 Mathematics Subject Classifications : 34C20, 34E20, 35S05

* Department of Mathematics and Physics, Faculty of Science and Technology, Kinki University, Higashi-Osaka 577, Japan.

Supported in part by Japanese Ministry of Education, Science and Culture under Grand-in-Aid for Scientific Research on Prior Area 04245235 and International Scientific Research Program 03044078

** Research Institute for Mathematical Sciences, Kyoto University, Kyoto 606, Japan. 


$$
\left(\frac{\partial^{2}}{\partial \tilde{x}^{2}}-Q(\tilde{x}) \frac{\partial^{2}}{\partial y^{2}}\right) \tilde{\varphi}_{B}=0
$$

and

$$
\left(\frac{\partial^{2}}{\partial x^{2}}-x \frac{\partial^{2}}{\partial y^{2}}\right) \varphi_{B}=0
$$

Hence the above result of [2] suggests that the microdifferential operator

$$
\left.\left(\frac{\partial^{2}}{\partial \tilde{x}^{2}}-Q(\tilde{x}) \frac{\partial^{2}}{\partial y^{2}}\right)\right|_{\tilde{x}=\tilde{x}(x)} T
$$

should be divisible by the differential operator

$$
\frac{\partial^{2}}{\partial x^{2}}-x \frac{\partial^{2}}{\partial y^{2}}
$$

from the right. In this paper we show that actually there exists an invertible microdifferential operator $S$ such that

$$
\left.\left(\frac{\partial^{2}}{\partial \tilde{x}^{2}}-Q(\tilde{x}) \frac{\partial^{2}}{\partial y^{2}}\right)\right|_{\tilde{x}=\tilde{x}(x)} T=S\left(\frac{\partial^{2}}{\partial x^{2}}-x \frac{\partial^{2}}{\partial y^{2}}\right) .
$$

We will determine the symbol of $S$. From the viewpoint of microdifferential calculus, we can say that (0.3) is the canonical form of the operators of the form

$$
\frac{\partial^{2}}{\partial \tilde{x}^{2}}-Q(\tilde{x}) \frac{\partial^{2}}{\partial y^{2}}
$$

around a simple zero of $Q$. Although this fact is well known (cf. [9]), we will give another proof, emphasizing the aspect of exact WKB analysis of differential operators. More precisely, we will find $S$ and $T$ which satisfy (0.4) under a special condition that $T$ preserves the Borel transformed WKB solutions. At the same time, we will generalize this reduction to the case where the potential $Q$ is a pre-Borel summable (see Definition 1.2) formal power series in $\eta^{-1}$ :

$$
\sum_{j=0}^{\infty} \eta^{-j} \boldsymbol{Q}_{j}(\tilde{\boldsymbol{x}})
$$

Such an operator naturally appears in the study of higher order differential equations and of monodromy preserving deformation of ordinary differential equations from the viewpoint of exact WKB analysis.

Another result of [2] is that if (0.1) has two simple turning points which are very close each other and connected by a Stokes curve emanating from one of these turning points, then (0.2) can be reduced to the "Weber-type" equation. We also generalize this to the case where the potential $Q$ is a pre-Borel summable formal power series.

In Section 1 we recall some notation. In Sections 2 and 3, we discuss the 
reduction to the Airy equation and to the Weber type equation.

The authors are indebted to Professor Kawai and Professor Takei for useful discussions.

\section{§1. Preliminaries}

We first fix notations and terminologies.

Definition 1.1. Let $f(\eta)$ be a formal power series in $\eta$ of the form

$$
f(\eta)=\exp \left(y_{0} \eta\right) \sum_{\lambda \in \Lambda_{+}(\alpha)} f_{\lambda} \eta^{-\lambda}
$$

where $\lambda$ runs over $\Lambda_{+}(\alpha)=\{\alpha+n \mid n=0,1, \ldots\}, \alpha \in \boldsymbol{R}$. Its Borel transform $f_{B}(y)$ is by definition

$$
f_{B}(y)=\sum_{\lambda \in \Lambda_{+}(\alpha)} \frac{f_{\lambda}}{\Gamma(1+\lambda)}\left(y+y_{0}\right)^{\lambda}
$$

We also need to consider the Borel transform of a formal series depending on a holomorphic parameter :

$$
f(x, \eta)=\exp \left(y_{0}(x) \eta\right) \sum_{\lambda \in \Lambda_{+}(\alpha)} f_{\lambda}(x) \eta^{-\lambda},
$$

where $f_{\lambda}(x)$ and $y_{0}(x)$ are holomorphic on an open set $U$ of C. Its Borel transform $f_{B}(x, y)$ is by definition

$$
f_{B}(x, y)=\sum_{\lambda \in \Lambda_{+}(\alpha)} \frac{f_{\lambda}(x)}{\Gamma(1+\lambda)}\left(y+y_{0}(x)\right)^{\lambda} .
$$

Definition 1. 2. The formal power series $f(x, \eta)$ given above is called preBorel summable on $U$ if for any compact set $K$ in $U$, there exist positive constants $A_{K}$ and $C_{K}$ such that

$$
\sup _{K}\left|f_{\lambda}(x)\right| \leq A_{K} C_{K}^{\lambda} \Gamma(1+\lambda)
$$

If $f(x, \eta)$ is pre-Borel summable, then $f_{B}(x, y)$ is a convergent series near $y=$ $-y_{0}(x)$ for each $x \in U$ and it defines a holomorphic function of $y$ near $y=$ $-y_{0}(x)$ for each $x \in U$. If $y_{0}(x)=0$ in addition, $f(x, \eta)$ is a symbol in the sense of [1], [9]. That is, $f(x, \eta)$ can be regarded as the total symbol of a microdifferential operator $f(x, \partial / \partial y)$.

Let $Q(x, \eta)$ be a formal power series in $\eta$ : 


$$
Q(x, \eta)=\sum_{j=0}^{\infty} \eta^{-j} Q_{j}(x),
$$

where $Q_{j}(j=0,1, \cdots)$ are holomorphic on an open set in C. We consider the following ordinary differential equation :

$$
\left(\frac{d^{2}}{d x^{2}}-\eta^{2} Q(x, \eta)\right) \varphi(x, \eta)=0
$$

A WKB solution of the equation is by definition a formal solution of the form

$$
\varphi(x, \eta)=\exp \left(\int^{x} S(x, \eta) d x\right)
$$

where $S$ is a formal power series :

$$
S(x, \eta)=\sum_{j=-1}^{+\infty} \eta^{-j} S_{j}(x)
$$

Such an $S$ is uniquely determined once we fix a branch of $S_{-1}(x)= \pm \sqrt{Q_{0}(x)}$. In fact, $S$ satisfies a Riccati-type equation

$$
\frac{\partial}{\partial x} S(x, \eta)+S(x, \eta)^{2}-\eta^{2} Q(x, \eta)=0
$$

and this reduces to the following recursive relations for $S_{j}$ 's :

$$
\begin{gathered}
S_{-1}^{2}=Q_{0}(x), \\
S_{j+1}=-\frac{1}{2 S_{-1}}\left(\sum_{k=0}^{j} S_{k} S_{j-k}+\frac{d S_{j}}{d x}-Q_{j+2}\right) .
\end{gathered}
$$

A zero point of $Q_{0}(x)$ is called a turning point of the equation. If it is a zero of order 1 , we call it a simple turning point.

Proposition 1.3. Let $Q(x, \eta)$ be a pre-Borel summable formal power series of $\eta$ :

$$
Q(x, \eta)=\sum_{j=0}^{\infty} \eta^{-j} Q_{j}(x)
$$

where $Q_{j}(j=0,1, \cdots)$ are holomorphic functions on an open set $U$ in $C$, and let $\varphi$ be a $W K B$ solution of the following equation:

$$
\left(\frac{d^{2}}{d x^{2}}-\eta^{2} Q(x, \eta)\right) \varphi(x, \eta)=0
$$

If there is no turning point in $U$, then $\varphi(x, \eta)$ is pre-Borel summable in $U$. 
This proposition is easily proved in a similar manner as Appendix A. 1 in [2]. Let $\varphi_{B}(x, y)$ denote the Borel transform of a WKB solution $\varphi(x, \eta)$ to (1. 1). Then $\varphi_{B}$ satisfies the following microdifferential equation :

$$
\left(\frac{\partial^{2}}{\partial \tilde{x}^{2}}-Q\left(\tilde{x}, \frac{\partial}{\partial y}\right) \frac{\partial^{2}}{\partial y^{2}}\right) \varphi_{B}=0
$$

Thus a part of exact WKB analysis can be described by the language of microdifferential calculus.

\section{$\S 2 . \quad$ Reduction to the Airy Equation}

We consider the following ordinary differential equation :

$$
\left(\frac{d^{2}}{d \tilde{x}^{2}}-\eta^{2} Q(\tilde{x}, \eta)\right) \tilde{\varphi}(\tilde{x}, \eta)=0
$$

Here $Q$ is a pre-Borel summable formal power series of $\eta$. We suppose that $\tilde{x}=$ 0 is a simple turning point. We try to reduce Equation (2.1) to the Airy equation with the large parameter $\eta$ :

$$
\left(\frac{d^{2}}{d x^{2}}-\eta^{2} x\right) \varphi(x, \eta)=0
$$

We take Borel transform of these equations and we will reduce the microdifferential operator

$$
\frac{\partial^{2}}{\partial \tilde{x}^{2}}-Q\left(\tilde{x}, \frac{\partial}{\partial y}\right) \frac{\partial^{2}}{\partial y^{2}}
$$

to the differential operator

$$
\frac{\partial^{2}}{\partial x^{2}}-x \frac{\partial^{2}}{\partial y^{2}}
$$

by some microdifferential operators and a change of variables that preserve WKB solutions. In order to state our theorem, we recall some notation concerned with microdifferential operators. For a microdifferential operator $L$, we denote its total symbol by $\sigma(L)$. To simplify the notation we denote $\sigma\left(\frac{\partial}{\partial x}\right)$ (resp. $\left.\sigma\left(\frac{\partial}{\partial y}\right)\right)$ by $\xi($ resp. $\eta$ ). Although a total symbol $a$ does not fix a microdifferential operator uniquely, there is a particular operation called a normal product of $a$, which is denoted as : $a$ : , so that the following holds (cf. [1]):

$$
: \sum_{h, j} a_{i, j}(x, y) \xi^{i} \eta^{j}:=\sum_{h, j} a_{i, j}(x, y) \frac{\partial^{i}}{\partial x^{i}} \frac{\partial^{j}}{\partial y^{j}} \text {. }
$$

Theorem 2.1. Let $Q(\tilde{x}, \eta)$ be a pre-Borel summable formal power series of 
$\eta$ on an open neighborhood of $\tilde{x}=0$ :

$$
Q(\tilde{x}, \eta)=\sum_{i=0}^{\infty} \eta^{-i} Q_{i}(\tilde{x})
$$

Suppose that $Q_{0}(\tilde{x})$ has a simple zero at $\tilde{x}=0$. Then, in a neighborhood of $\tilde{x}=0$, there exist another holomorphic coordinate $x(\tilde{x})$ with $x(0)=0$ and invertible microdifferential operators $S$ and $T$ of the forms

$$
\begin{aligned}
& S=: g^{\prime}(x)^{\frac{5}{2}}\left(1+\frac{\partial r(x, \eta)}{\partial x}\right)^{\frac{3}{2}} \exp (r(x, \eta) \xi):, \\
& T=: g^{\prime}(x)^{\frac{1}{2}}\left(1+\frac{\partial r(x, \eta)}{\partial x}\right)^{-\frac{1}{2}} \exp (r(x, \eta) \xi):
\end{aligned}
$$

for which the following holds :

$$
\left.\left(\frac{\partial^{2}}{\partial \tilde{x}^{2}}-Q\left(\tilde{x}, \frac{\partial}{\partial y}\right) \frac{\partial^{2}}{\partial y^{2}}\right)\right|_{\tilde{x}=g(x)} T=S\left(\frac{\partial^{2}}{\partial x^{2}}-x \frac{\partial^{2}}{\partial y^{2}}\right) .
$$

Here $\tilde{x}=g(x)$ denotes the inverse function of $x=x(\tilde{x})$ on a neighborhood of $x=0$ and $r(x, \eta)=\sum_{j=1}^{\infty} r_{j}(x) \eta^{-j}$ is a symbol of order -1 .

Remark. To see the relation of our result and Proposition 2. 3 of [2], we note the following :

$$
: \exp (r \xi):\left.\varphi(x)\right|_{x=x(\tilde{x})}=\varphi(x(\tilde{x})+r)
$$

We also note that $\exp (r \xi)$ is a symbol of order 0 and hence $S$ and $T$ in Theorem 2. 1 are of order 0 .

Let $x=x(\tilde{x})$ be a holomorphic function satisfying

$$
Q_{0}(\tilde{x})=\left(\frac{d x(\tilde{x})}{d \tilde{x}}\right)^{2} x(\tilde{x})
$$

Such a function uniquely exists and satisfies $\frac{\partial x}{\partial \tilde{x}}(0) \neq 0$ since $Q_{0}$ has a simple zero at the origin. Then we find

$$
\begin{aligned}
\frac{\partial^{2}}{\partial \tilde{x}^{2}}-Q\left(\tilde{x}, \frac{\partial}{\partial y}\right) \frac{\partial^{2}}{\partial y^{2}}= & \frac{1}{\left(g^{\prime}\right)^{2}}\left(\frac{\partial^{2}}{\partial x^{2}}-x \frac{\partial^{2}}{\partial y^{2}}-\frac{g^{\prime \prime}}{g^{\prime}} \frac{\partial}{\partial x}\right. \\
& \left.-\left(g^{\prime}\right)^{2} \sum_{i=1}^{\infty} Q_{i}(g(x))\left(\frac{\partial}{\partial y}\right)^{2-i}\right) .
\end{aligned}
$$

Here $\tilde{x}=g(x)$ is the inverse function of $x=x(\tilde{x})$ on a neighborhood of $x=0$ and $g^{\prime}, g^{\prime \prime}$ denote $\frac{\partial g}{\partial x}, \frac{\partial^{2} g}{\partial x^{2}}$ respectively. To prove Theorem 2. 1 , it is enough to show the following :

Proposition 2.2. Let $A$ and $B$ denote respectively the following microdiffer- 
ential operators :

$$
\begin{gathered}
A=\frac{\partial^{2}}{\partial x^{2}}-x \frac{\partial^{2}}{\partial y^{2}}-\frac{g^{\prime \prime}}{g^{\prime}} \frac{\partial}{\partial x}-\left(g^{\prime}\right)^{2} \sum_{i=1}^{\infty} Q_{i}(g(x))\left(\frac{\partial}{\partial y}\right)^{2-\imath}, \\
B=\frac{\partial^{2}}{\partial x^{2}}-x \frac{\partial^{2}}{\partial y^{2}} .
\end{gathered}
$$

Here $Q_{i}(i=1,2, \cdots)$ and $g$ are given as above. Then there exist invertible microdifferential operators $X$ and $Y$ defined on a neighborhood of $(x, y, \xi, \eta)=(0$, $0 ; 0,1)$, of the forms

$$
X=: g^{\prime}(x)^{\frac{1}{2}}\left(1+\frac{\partial r(x, \eta)}{\partial x}\right)^{-\frac{1}{2}} \exp (r(x, \eta) \xi):
$$

and

$$
Y=: g^{\prime}(x)^{\frac{1}{2}}\left(1+\frac{\partial r(x, \eta)}{\partial x}\right)^{\frac{3}{2}} \exp (r(x, \eta) \xi):
$$

respectively, for which the following holds :

$$
A X=Y B
$$

Here $r(x, \eta)=\sum_{j=1}^{\infty} r_{j}(x) \eta^{-j}$ is a symbol of microdifferential operator of order -1 .

Proof. Let us calculate the total symbols of both members of (2.2). We assume that $X$ is written in the form

$$
X=: C(x, \eta) \exp (r(x, \eta) \xi):
$$

with some symbols $r$ and $C$ which are independent of $y$ and $\xi$. We assume further that $Y$ has the form

$$
Y=: C_{1}(x, \xi, \eta) \exp (r(x, \eta) \xi):,
$$

where $C_{1}$ does not contain $y$. Under these assumptions, we will determine $r, C$ and $C_{1}$ so that (2.2) holds. By the composition rule of total symbols of microdifferential operators, we have

$$
\begin{aligned}
\sigma(A X)= & \sigma(A) \sigma(X)+\frac{\partial \sigma(A)}{\partial \xi} \frac{\partial \sigma(X)}{\partial x}+\frac{1}{2 !} \frac{\partial^{2} \sigma(A)}{\partial \xi^{2}} \frac{\partial^{2} \sigma(X)}{\partial x^{2}}+\cdots \\
= & \left(C\left(1+\frac{\partial r}{\partial x}\right)^{2} \xi^{2}+\left(2\left(1+\frac{\partial r}{\partial x}\right) \frac{\partial C}{\partial x}+\left(\frac{\partial^{2} r}{\partial x^{2}}-\frac{g^{\prime \prime}}{g^{\prime}}\left(1+\frac{\partial r}{\partial x}\right)\right) C\right) \xi\right. \\
& \left.+\frac{\partial^{2} C}{\partial x^{2}}-\frac{g^{\prime \prime}}{g^{\prime}} \frac{\partial C}{\partial x}-x \eta^{2} C-g^{\prime}{ }^{2} \sum_{i=1}^{\infty} Q_{i}(g(x)) \eta^{2-i} C\right) \exp (r \xi)
\end{aligned}
$$


and

$$
\sigma(Y B)=\left(C_{1} \xi^{2}-\left(C_{1}(x+r)+\frac{\partial C_{1}}{\partial \xi}\right) \eta^{2}\right) \exp (r \xi)
$$

Comparing the both right-hand sides, we see that $C_{1}$ must be independent of $\xi$ and we have the following system of differential equations for $C, C_{1}$ and $r$ :

$$
\begin{gathered}
C\left(1+\frac{\partial r}{\partial x}\right)^{2}=C_{1}, \\
2\left(1+\frac{\partial r}{\partial x}\right) \frac{\partial C}{\partial x}+\left(\frac{\partial^{2} r}{\partial x^{2}}-\frac{g^{\prime \prime}}{g^{\prime}}\left(1+\frac{\partial r}{\partial x}\right)\right) C=0, \\
\frac{\partial^{2} C}{\partial x^{2}}-\frac{g^{\prime \prime}}{g^{\prime}} \frac{\partial C}{\partial x}-\left(x \eta^{2}+g^{\prime} \sum_{i=1}^{\infty} Q_{i}(g(x)) \eta^{2-i}\right) C=-C_{1}(x+r) \eta^{2} .
\end{gathered}
$$

Equation (2.4) is rewritten in the form

$$
\frac{\partial C}{\partial x}=\frac{1}{2}\left(\frac{g^{\prime \prime}}{g^{\prime}}-\frac{\frac{\partial^{2} \tilde{r}}{\partial x^{2}}}{\frac{\partial \tilde{r}}{\partial x}}\right) C,
$$

where we set $\tilde{r}=x+r$. If we suppose that $\tilde{r}$ is given, this equation can be solved easily :

$$
C=\left(\frac{\partial g}{\partial x}\right)^{\frac{1}{2}}\left(\frac{\partial \tilde{r}}{\partial x}\right)^{-\frac{1}{2}}=\left(\frac{\partial \tilde{r}}{\partial \tilde{x}}\right)^{-\frac{1}{2}}
$$

is a solution. Thus $C$ and $C_{1}$ can be eliminated in (2.5) and a single equation for $\tilde{r}$ is obtained. Using the definition of $x=x(\tilde{x})$, we rewrite it in the form

$$
\sum_{i=0}^{\infty} Q_{i}(\tilde{x}) \eta^{2-i}=\left(\frac{\partial \tilde{r}}{\partial \tilde{x}}\right)^{2} \tilde{m} \eta^{2}-\frac{1}{2}\{\tilde{r} ; \tilde{x}\}
$$

Here $\{\tilde{r} ; \tilde{x}\}$ denotes the Schwarzian derivative :

$$
\frac{\frac{\partial^{3} \tilde{r}}{\partial \tilde{x}^{3}}}{\frac{\partial \tilde{r}}{\partial \tilde{x}}}-\frac{3}{2}\left(\frac{\frac{\partial^{2} \tilde{r}}{\partial \tilde{x}^{2}}}{\frac{\partial \tilde{r}}{\partial \tilde{x}}}\right)^{2} .
$$

We suppose that $\tilde{r}$ has the following form :

$$
\tilde{r}=\sum_{i=0}^{\infty} x_{i}(\tilde{x}) \eta^{-i}
$$

We can easily write down in a similar manner as in $\S$ A. 2 in [2] the relations which such $x_{i}$ 's should satisfy formally. Denoting $d x_{j} / d \tilde{x}$ etc. by $x_{j}^{\prime}$ etc., we 
have the following :

$$
\begin{gathered}
Q_{0}(\tilde{x})=x_{0}^{\prime}(\tilde{x})^{2} x_{0}(\tilde{x}), \\
x_{0}^{\prime}\left(2 x_{0} \frac{d}{d \tilde{x}}+x_{0}^{\prime}\right) x_{1}=Q_{1}(\tilde{x}),
\end{gathered}
$$

(2. $7 . n+2)$

$$
\begin{gathered}
x_{0}^{\prime}\left(2 x_{0} \frac{d}{d \tilde{x}}+x_{0}^{\prime}\right) x_{n+2}=\frac{1}{2} \sum_{k+l+\mu=n} \sum_{\mu_{1}+}(-1)^{l}\left(x_{0}^{\prime}\right)^{-l-1} x_{k}^{\prime \prime \prime} x_{\mu_{1}+1}^{\prime} \cdots x_{\mu_{l}+1}^{\prime} \\
\quad-\frac{3}{4} \sum_{k_{1}+k_{2}+l+\mu=n} \sum_{\substack{\mu_{1}+\cdots+\mu_{l}=\mu\\
}}(-1)^{l}(l+1)\left(x_{0}^{\prime}\right)^{-l-2} x_{k_{1}}^{\prime \prime} x_{k_{2}}^{\prime \prime} x_{\mu_{1}+1}^{\prime} \cdots x_{\mu_{l}+1}^{\prime} \\
-\sum_{k_{1}+k_{2}+l=n+2} x_{k_{1}}^{\prime} x_{k_{2}}^{\prime} x_{l}+Q_{n+2}(\tilde{x}) .
\end{gathered}
$$

Here $n=0,1,2, \cdots$. If we set $x_{0}=x$, then (2.7.0) trivially holds, for it is the same as the definition of $x=x(\tilde{x})$. Now we can determine holomorphic functions $x_{j}(j=1,2, \cdots)$ inductively from (2.7.j), since the characteristic exponent of the differential operator

$$
2 x_{0} \frac{d}{d \tilde{x}}+x_{0}^{\prime}
$$

is a non-integer $-1 / 2$ and the right-hand side of (2.7. $j)(j \geq 1)$ is holomorphic near $\tilde{x}=0$ at each stage of induction. Hence we have obtained a formal solution to $(2.3)-(2.5)$.

We have to show that those $r, C$ and $C_{1}$ are actually symbols of microdifferential operators. It suffices to see that $\tilde{r}$ is a symbol. What we need is the following estimate : Let $U$ be a sufficiently small neighborhood of the origin in $C$. For every compact set $K$ in $U$, there exists a positive constant $M$ so that

$$
\sup _{\tilde{x} \in K}\left|x_{n}(\tilde{x})\right| \leq n ! M^{n+1}
$$

holds for every $n=0,1,2, \cdots$.

We note that our construction of $x_{j}^{\prime}$ 's looks almost the same as the construction of $q_{j}$ 's of $\S$ A. 2 in [2]. There are two essentially different points between those constructions. The first is the appearance of the term $Q_{n+2}$ in (2. 7. $n+2)$. Here we note that $Q=\sum_{j=0}^{\infty} \eta^{-j} Q_{j}$ is pre-Borel summable by the assumption. The second is the fact that $x_{1}$ does not vanish identically in our case while $q_{1} \equiv 0$ in [2]. Taking them into account, we can make a version-up of the

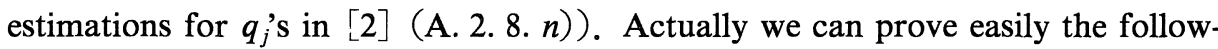
ing :

Lemma 2.3. Let $\rho$ be a sufficiently small positive number. There exist 
positive constants $L$ and $M$ for which

$$
\sup _{|\tilde{x}| \leq \rho-\varepsilon}\left|\boldsymbol{x}_{n}(\tilde{x})\right| \leq n ! L M^{n-1} \varepsilon^{-n}
$$

and

$$
\sup _{|\tilde{x}| \leq \rho-\varepsilon}\left|x_{n}^{\prime}(\tilde{x})\right| \leq n ! L M^{n-1} \varepsilon^{-n}
$$

hold for every $\varepsilon<\rho$ and for every $n=1,2, \cdots$.

This lemma implies that $r$ is a symbol. Hence $C$ and $C_{1}$ are also symbols. Invertibility of the microdifferential operators $X$ and $Y$ is clear since the principal parts do not vanish near $(x, y ; \xi, \eta)=(0,0 ; 0,1)$.

Remark. Let us consider the following second order differential operator :

$$
\frac{d^{2}}{d x^{2}}+\eta P(x, \eta) \frac{d}{d x}+\eta^{2} Q(x, \eta) .
$$

Here $P(x, \eta)$ and $Q(x, \eta)$ are formal power series of $\eta^{-1}$. It is well known that the first-order term can be eliminated easily :

$$
\begin{aligned}
& \left(\frac{d^{2}}{d x^{2}}+\eta P(x, \eta) \frac{d}{d x}+\eta^{2} Q(x, \eta)\right) \exp \left(-\frac{\eta}{2} \int P(x, \eta) d x\right) \\
& \quad=\exp \left(-\frac{\eta}{2} \int P(x, \eta) d x\right)\left(\frac{d^{2}}{d x^{2}}+\eta^{2} Q(x, \eta)-\frac{1}{4} \eta^{2} P(x, \eta)^{2}-\frac{1}{2} \eta \frac{d P}{d x}\right)
\end{aligned}
$$

Thus all the results given so far equally hold for operators with first order terms.

\section{§ 3. Reduction to the Weber Type Equation}

Let us consider the equation

$$
\left(\frac{d^{2}}{d \tilde{x}^{2}}-\eta^{2} Q(\tilde{x})\right) \tilde{\phi}(\tilde{x}, \eta)=0
$$

where $Q$ is a holomorphic function defined in an open neighborhood $U$ of the origin 0 of C. It is shown in [2] that if Equation (3.1) has exactly two turning points in $U$ and they are connected by a Stokes curve (cf. [10]) in $U$ emanating from one of them, then (3.1) can be reduced to the following Weber type equation : 


$$
\left(\frac{d^{2}}{d x^{2}}-\eta^{2}\left(E(\eta)-\frac{x^{2}}{4}\right)\right) \phi(x, \eta)=0 .
$$

In this section, we generalize this result to the case where $Q$ is a pre-Borel summable formal power series in $\eta$ with holomorphic coefficients. As in the previous section, we take Borel transformation and we state our theorem in the form of equivalence of microdifferential operators :

Theorem 3.1. Let $Q(\tilde{x}, \eta)$ be a pre-Borel summable formal power series of $\eta$ defined on an open neighborhood $U$ of the origin 0 of $\mathbf{C}$ :

$$
Q(\tilde{x}, \eta)=\sum_{i=0}^{\infty} \eta^{-i} Q_{i}(\tilde{x})
$$

Suppose that $Q_{0}(\tilde{x})$ has exactly two simple zeros $p_{0}$ and $p_{1}$ in $U$, and suppose further that $p_{0}$ and $p_{1}$ are connected by a curve $\Gamma$ in $U$ defined by

$$
\operatorname{Im} \int_{P_{0}}^{\tilde{x}} \sqrt{Q_{0}(\tilde{x})} d \tilde{x}=0 .
$$

Then there exist another holomorphic coordinate $x=x(\tilde{x})$ near the curve $\Gamma$ and microdifferential operators $E, S$ and $T$ of order 0 and of the forms

$$
\begin{gathered}
E=: \sum_{j=0}^{\infty} E_{j} \eta^{-j}:, \\
S=: g^{\prime}(x)^{\frac{5}{2}}\left(1+\frac{\partial r(x, \eta)}{\partial x}\right)^{\frac{3}{2}} \exp (r(x, \eta) \xi):,
\end{gathered}
$$

where $\tilde{x}=g(x)$ is the inverse function of $x=x(\tilde{x})$, and

$$
T=: g^{\prime}(x)^{\frac{1}{2}}\left(1+\frac{\partial r(x, \eta)}{\partial x}\right)^{-\frac{1}{2}} \exp (r(x, \eta) \xi):
$$

so that the following holds:

(a) Each $E_{j}$ is a constant $(j=1,2, \cdots)$ and $E_{0}$ is a real positive constant given by

$$
E_{0}=\frac{1}{\pi} \int_{P_{0}}^{p_{1}} \sqrt{Q_{0}(\tilde{x})} d \tilde{x} .
$$

(b) $x\left(p_{0}\right)=-2 \sqrt{E_{0}}$ and $x\left(p_{1}\right)=2 \sqrt{E_{0}}$.

(c) $r(x, \eta)=\sum_{j=1}^{\infty} r_{j}(x) \eta^{-j}$ is a symbol of order -1 .

(d) $S$ and $T$ are invertible and satisfy the relation

$$
\left.\left(\frac{\partial^{2}}{\partial \tilde{x}^{2}}-Q\left(\tilde{x}, \frac{\partial}{\partial y}\right) \frac{\partial^{2}}{\partial y^{2}}\right)\right|_{\tilde{x}=g(x)} T=S\left(\frac{\partial^{2}}{\partial x^{2}}-\left(E-\frac{x^{2}}{4}\right) \frac{\partial^{2}}{\partial y^{2}}\right) .
$$

It is shown in [2] that if $E_{0}$ is chosen as (3.2), then there exists a holomorphic 
coordinate $x=x(\tilde{x})$ on a neighborhood of $\Gamma$ so that $x\left(p_{0}\right)=-2 \sqrt{E_{0}}, x\left(p_{1}\right)=$ $2 \sqrt{E_{0}}$ and

$$
Q_{0}(\tilde{x})=x^{\prime 2}\left(E_{0}-\frac{x^{2}}{4}\right)
$$

hold. In the coordinates $(x, y)$, we have

$$
\begin{aligned}
\frac{\partial^{2}}{\partial \tilde{x}^{2}}-Q\left(\tilde{x}, \frac{\partial}{\partial y}\right) \frac{\partial^{2}}{\partial y^{2}}=\frac{1}{g^{\prime 2}}( & \frac{\partial^{2}}{\partial x^{2}}-\left(E_{0}-\frac{x^{2}}{4}\right) \frac{\partial^{2}}{\partial y^{2}} \\
& \left.-\frac{g^{\prime \prime}}{g^{\prime}} \frac{\partial}{\partial x}-g^{\prime 2} \sum_{j=1}^{\infty} Q_{j}(g(x))\left(\frac{\partial}{\partial y}\right)^{2-j}\right) .
\end{aligned}
$$

Now we set

$$
A=\frac{\partial^{2}}{\partial x^{2}}-\left(E_{0}-\frac{x^{2}}{4}\right) \frac{\partial^{2}}{\partial y^{2}}-\frac{g^{\prime \prime}}{g^{\prime}} \frac{\partial}{\partial x}-g^{\prime}{ }^{2} \sum_{j=1}^{\infty} Q_{j}(g(x))\left(\frac{\partial}{\partial y}\right)^{2-j} .
$$

To prove Theorem 3. 1, it suffices to show the following proposition :

Proposition 3.2. Let $Q, A, g$ be as above. Then there exist microdifferential operators $E, X$ and $Y$ of order 0 and of the forms

$$
\begin{gathered}
E=: \sum_{j=0}^{\infty} E_{j} \eta^{-j}:, \\
X=: g^{\prime}(x)^{\frac{1}{2}}\left(1+\frac{\partial r(x, \eta)}{\partial x}\right)^{-\frac{1}{2}} \exp (r(x, \eta) \xi):
\end{gathered}
$$

and

$$
Y=: g^{\prime}(x)^{\frac{1}{2}}\left(1+\frac{\partial r(x, \eta)}{\partial x}\right)^{\frac{3}{2}} \exp (r(x, \eta) \xi):
$$

respectively, so that the following holds:

(a) Each $E_{j}$ is a constant.

(b ) $\quad r(x, \eta)=\sum_{j=1}^{\infty} r_{j}(x) \eta^{-j}$ is a symbol of order -1 .

(c) Let $B$ denote the following microdifferential operator:

$$
B=\frac{\partial^{2}}{\partial x^{2}}-\left(E-\frac{x^{2}}{4}\right) \frac{\partial^{2}}{\partial y^{2}} .
$$

Then $X$ and $Y$ are invertible and satisfy the relation

$$
A X=Y B
$$

Proof. We suppose $X$ and $Y$ are written respectively in the form

$$
X=: C(x, \eta) \exp (r(x, \eta) \xi):
$$


and

$$
Y=: C_{1}(x, \xi, \eta) \exp (r(x, \eta) \xi):,
$$

with some symbols $C, C_{1}$ of order 0 and $r$ of order -1 . Similar calculation as in the proof of Proposition 2. 2 shows that if (3.4) holds then $C, C_{1}$ and $r$ should satisfy the relations

$$
\begin{gathered}
C\left(1+\frac{\partial r}{\partial x}\right)^{2}=C_{1}, \\
2\left(1+\frac{\partial r}{\partial x}\right) \frac{\partial C}{\partial x}+\left(\frac{\partial^{2} r}{\partial x^{2}}-\frac{g^{\prime \prime}}{g^{\prime}}\left(1+\frac{\partial r}{\partial x}\right)\right) C=0 \\
\frac{\partial^{2} C}{\partial x^{2}}-\frac{g^{\prime \prime}}{g^{\prime}} \frac{\partial C}{\partial x}-\left(\left(E_{0}-\frac{x^{2}}{4}\right) \eta^{2}+g^{\prime 2} \sum_{i=1}^{\infty} Q_{i}(g(x)) \eta^{2-i}\right) C \\
=-C_{1}\left(\sum_{j=0}^{\infty} E_{j} \eta^{-j}-\frac{1}{4}(x+r)^{2}\right) \eta^{2} .
\end{gathered}
$$

We see that (3.5) and (3.6) have the same form as (2.3) and (2.4), respectively. Hence

$$
C=\left(\frac{\partial \tilde{r}}{\partial \tilde{x}}\right)^{-\frac{1}{2}}
$$

satisfies (3.6), where we set $\tilde{r}=x+r$. Thus we can eliminate $C$ and $C_{1}$ in (3.7) and have a single equation for $\tilde{r}$. We rewrite it by using (3.3), in the form

$$
\sum_{j=0}^{\infty} Q_{j}(\tilde{x}) \eta^{2-j}=\left(\frac{\partial \tilde{r}}{\partial \tilde{x}}\right)^{2}\left(\sum_{j=0}^{\infty} E_{j} \eta^{-j}-\frac{\tilde{r}^{2}}{4}\right) \eta^{2}-\frac{1}{2}\{\tilde{r} ; \tilde{x}\} .
$$

Here $\{\tilde{r} ; \tilde{x}\}$ denotes the Schwarzian derivative. We suppose that $\tilde{r}$ has the following form :

$$
\tilde{r}=\sum_{i=0}^{\infty} x_{i}(\tilde{x}) \eta^{-i}
$$

Then we can write down in a similar way as in $\S 3$ in [2] the relations which $x_{j}$ 's should satisfy formally :

$$
Q_{0}(\tilde{x})=x_{0}^{\prime 2}\left(E_{0}-\frac{x_{0}^{2}}{4}\right)
$$

$$
\left(\left(E_{0}-\frac{x_{0}^{2}}{4}\right) \frac{d}{d \tilde{x}}-\frac{1}{4} x_{0}^{\prime} x_{0}\right) x_{1}=\frac{1}{2}\left(\frac{Q_{1}(\tilde{x})}{x_{0}^{\prime}}-x_{0}^{\prime} E_{1}\right),
$$

(3. $9 . n+2)$

$$
\left(\left(E_{0}-\frac{x_{0}^{2}}{4}\right) \frac{d}{d \tilde{x}}-\frac{1}{4} x_{0}^{\prime} x_{0}\right) x_{n+2}=\frac{1}{2}\left(\frac{Q_{n+2}(\tilde{x})}{x_{0}^{\prime}}-x_{0}^{\prime} E_{n+2}\right)+R_{n} \text {. }
$$

Here we denote $d x_{j} / d \tilde{x}$ by $x_{j}^{\prime}$ etc. and $R_{n}(n=0,1,2, \cdots)$ is given by 


$$
\begin{aligned}
R_{n}= & -\frac{1}{2} \sum_{\substack{k_{1}+k_{2}+l=n+2 \\
k_{1}, k_{2}, l<n+2}} \frac{x_{k_{1}}^{\prime} x_{k_{2}}^{\prime}}{x_{0}^{\prime}} E_{l}+\frac{1}{8} \sum_{\substack{k_{1}+k_{2}+l_{1}+l_{2}=n+2 \\
k_{1}, k_{2}, l_{1}, l_{2}<n+2}} \frac{x_{k_{1}}^{\prime} x_{k_{2}}^{\prime}}{x_{0}^{\prime}} x_{l_{1}} x_{l_{2}} \\
& +\frac{1}{4} \sum_{k+J+\mu=n} \sum_{\mu_{1}+\cdots+\mu_{l}=\mu}(-1)^{l} \frac{x_{k}^{\prime \prime \prime}}{\left(x_{0}^{\prime}\right)^{l+2}} x_{\mu_{1}+1}^{\prime} \cdots x_{\mu_{l}+1}^{\prime} \\
& -\frac{3}{8} \sum_{k+\jmath+l+\mu=n} \sum_{\mu_{1}+\cdots+\mu_{l}=\mu}(-1)^{l}(l+1) \frac{x_{k_{1}}^{\prime \prime} x_{k_{2}}^{\prime \prime}}{\left(x_{0}^{\prime}\right)^{l+3}} x_{\mu_{1}+1}^{\prime} \cdots x_{\mu_{l}+1}^{\prime} .
\end{aligned}
$$

Here $n=0,1,2, \cdots$. If we set $x_{0}=x$, then (2. 7.0) trivially holds (cf. (3.3)). We note that $R_{n}$ depends only on $x_{i}$ and $E_{i}$ for $i=0, \cdots, n+1$. Equation (3.9. $j$ ) has a unique holomorphic solution $x_{j}$ on a common neighborhood of $\Gamma$ provided the constant $E_{j}$ is chosen appropriately, for we know the following lemma (see the proof of Lemma 3.4 in [2]) :

Lemma 3. 3. Let $E_{0}$ be a positive constant and $\kappa$ a constant. Let $\mathbb{W}(\rho)(\rho>$ 0) denote the following compact set:

$$
W(\rho)=\left\{x \in \mathbb{C}|| x-s \mid \leq \rho \text { for some } s \in\left[-2 \sqrt{E_{0}}, 2 \sqrt{E_{0}}\right]\right\}
$$

Let $w$ be a holomorphic function defined on a neighborhood of $W(\rho)$. Then the differential equation

$$
\left(\left(E_{0}-\frac{x^{2}}{4}\right) \frac{d}{d x}-\frac{x}{4}\right) v=-\frac{1}{2} \kappa+w(x)
$$

has a unique holomorphic solution on the neighborhood if and only if

$$
\kappa=\frac{2 \int_{-2 \sqrt{E_{0}}}^{2 \sqrt{E_{0}}}\left(E_{0}-\frac{s^{2}}{4}\right)^{-\frac{1}{2}} w(x) d s}{\int_{-2 \sqrt{E_{0}}}^{2 \sqrt{E_{0}}}\left(E_{0}-\frac{s^{2}}{4}\right)^{-\frac{1}{2}} d s} .
$$

We write (3.9.j) in the coordinate $x=x_{0}(\tilde{x})$. Then by Lemma 3. 3, we can determine $x_{j}$ and $E_{j}$ successively. Thus we have found a formal solution $(\tilde{r}, E=$ $\sum E_{j} \eta^{-j}$ ) of (3.8). We have to see that $\tilde{r}$ and $E$ are actually symbols. In other words, we must show that $\tilde{r}$ and $E$ are pre-Borel summable. What we have to see is the following estimates for $x_{j}$ 's and for $E_{j}$ 's :

(i) Let $U$ be an open neighborhood of $\Gamma$ so that each $x_{j}$ is holomorphic on $U$. For every compact set $K$ in $U$, there exists a positive constant $M$ for which

$$
\sup _{\tilde{x} \in K}\left|x_{n}(\tilde{x})\right| \leq n ! M^{n+1}
$$


holds for every $n=0,1,2, \cdots$.

(ii) There exists a positive constant $M_{1}$ for which

$$
\left|E_{n}\right| \leq n ! M_{1}^{n+1}
$$

holds for every $n=0,1,2, \cdots$.

These estimates can be proved in a similar way as in preceding section and $\S \mathrm{A} 2$ of $[2]$. We only note here the following lemma which is crucial to have the above estimates :

Lemma 3.4. Let $E_{0}, W(\rho)$ and $w$ be as in Lemma 3. 3 and $\kappa$ the constant given by (3.11). Let $v$ be the unique holomorphic solution of (3.10). Then there exists a positive constant $L$ so that

$$
\begin{aligned}
& \sup _{x \in W(\rho)}|v(x)| \leq L \\
& \sup _{x \in W(\rho)}|w(x)|, \\
& \sup _{x \in W(\rho)}\left|\frac{d v(x)}{d x}\right| \leq \frac{L}{\rho} \sup _{x \in W(\rho)}|w(x)|
\end{aligned}
$$

for every sufficiently small $\rho$.

Proof. At first, we remark that the following holds trivially :

$$
|\kappa| \leq 2 \sup _{-2 \sqrt{E_{0}} \leq x \leq 2 \sqrt{E_{0}}}|w(x)| .
$$

We divide $W(\rho)$ into three subsets : $W(\rho)=D_{0} \cup D_{1} \cup D$, where

$$
\begin{aligned}
& D_{0}=\left\{x|| x+2 \sqrt{E_{0}} \mid \leq \rho\right\} \\
& D_{1}=\left\{x|| x-2 \sqrt{E_{0}} \mid \leq \rho\right\},
\end{aligned}
$$

and

$$
D=W(\rho)-\left(D_{0} \cup D_{1}\right)
$$

We set $y=x / 2+\sqrt{E_{0}}$. The holomorphic solution $v$ is written in the form

$$
\begin{aligned}
v(x)=2\left(y\left(2 \sqrt{E_{0}}-y\right)\right)^{-\frac{1}{2}} \int_{0}^{y}\left(z\left(2 \sqrt{E_{0}}-z\right)\right)^{-\frac{1}{2}} \\
\quad \times\left.\left(-\frac{1}{2} \kappa+w\left(2\left(z-\sqrt{E_{0}}\right)\right)\right) d z\right|_{y=x / 2+\sqrt{E_{0}}} .
\end{aligned}
$$

Combining this with (3.12) yields 


$$
\sup _{x \in D_{0}}|v(x)| \leq 8 \sup _{|y| \leq \frac{o}{2}}\left|\left(2 \sqrt{E_{0}}-y\right)^{-1}\right| \sup _{x \in W(\rho)}|w(x)| .
$$

Thus we obtain

$$
\sup _{x \in D_{0}}|v(x)| \leq L_{1} \sup _{x \in W(\rho)}|w(x)|
$$

for some positive constant $L_{1}$. Since $v$ satisfies (3.10), we find

$$
y \frac{d v}{d y}=\frac{2 w-\kappa}{2 \sqrt{E_{0}}-y}-\frac{\sqrt{E_{0}}-y}{2 \sqrt{E_{0}}-y} v .
$$

Hence there exists a positive constant $L_{2}$ such that

$$
\sup _{|y| \leq \frac{\rho}{2}}\left|y \frac{d v}{d y}\right| \leq L_{2} \sup _{x \in W(\rho)}|w(x)|
$$

By the Schwarz lemma, we have

$$
\sup _{|y| \leq \frac{\rho}{2}}\left|\frac{d v}{d y}\right| \leq \frac{2 L_{2}}{\rho} \sup _{x \in W(\rho)}|w(x)| .
$$

Thus we have the following inequality :

$$
\sup _{x \in D_{0}}\left|\frac{d v}{d x}\right| \leq \frac{L_{2}}{\rho} \sup _{x \in W(\rho)}|w(x)| .
$$

Similar argument as above shows that there exist positive constants $L_{3}$ and $L_{4}$ for which

$$
\sup _{x \in D_{1}}|v(x)| \leq L_{3} \sup _{x \in W(\rho)}|w(x)|
$$

and

$$
\sup _{x \in D_{1}}\left|\frac{d v}{d x}\right| \leq \frac{L_{4}}{\rho} \sup _{x \in W(\rho)}|w(x)|
$$

hold. For the estimations of $|v|$ and $|d v / d x|$ in $D$, we easily see that there exist positive constants $L_{5}$ and $L_{6}$ so that

$$
\sup _{x \in D}|v(x)| \leq L_{5} \sup _{x \in W(o)}|w(x)|
$$

and

$$
\sup _{x \in D}\left|\frac{d v}{d x}\right| \leq \frac{L_{6}}{\rho} \sup _{x \in W(\rho)}|w(x)| .
$$

The former can be proved in a similar manner as above and the latter is trivial since $|y| \geq \rho / 2$ in $D$. The proof of Lemma 3.4 completes if we set $L$ 
$=\max \left\{L_{j} \mid j=1, \cdots, 6\right\}$.

Thus we have obtained microdifferential operators $X$ and $Y$ which satisfy the condition of Proposition 3. 2, for invertibility of those operators is clear.

\section{References}

[1] Aoki, T., Symbols and formal symbols of pseudodifferential operators, Adv. Stud. in Pure Math., 4 (1984), 181-208.

[2] Aoki, T., Kawai, T. and Takei, Y., The Bender-Wu analysis and the Voros theory, ICM-90 Satellite Conference Proceedings, Special Functions, Springer-Verlag, (1991), 1-29.

[3] - New turning points in the exact WKB analysis for higher-order ordinary differential equations, RIMS 853 , preprint.

[4] Bender, C. M. and Wu, T. T., Anharmonic oscillator, Phys. Rev., 184 (1969), 1231-1260.

[5] Candelpergher, B., Nosmas, C. and Pham, F., Résurgence et développements semiclassique, to appear.

[6] Delabaere, E. and Dillinger, H., Contribution à la résurgence quantique-Résurgence de Voros et fonction spectrale de Jost, Thèse de Doctorat, Université de Nice-Sophia-Antipolis, 1991.

[7] Ecalle, J., Les fonctions résurgentes, 1-3, Publ. Math. Orsay, Univ. Paris-Sud, 1981, 1985.

[8] Pham, F., Resurgence, quantized canonical transformations, and multi-instanton expansions, Algebraic Analysis, II, Academic Press, (1988), 699-726.

[9] Sato, M., Kawai, T. and Kashiwara, M., Microfunctions and pseudo-differential equations, Lecture Notes in Math., Springer, 287 (1973), 265-529.

[10] Voros, A., The return of the quartic oscillator-The complex WKB method, Ann. Inst. Henri Poincaré, 39 (1983), 211-338. 
